EPJ Web of Conferences 64, 05001 (2014)

DOI: $10.1051 /$ epjconf/ 20146405001

(C) Owned by the authors, published by EDP Sciences, 2014

\title{
MHD Simulations of Magnetospheric Accretion, Ejection and Plasma-field Interaction
}

\author{
M. M. Romanova ${ }^{1, a}$, R. V. E. Lovelace ${ }^{1,}$, M. Bachetti2, A. A. Blinova ${ }^{1,}$, A. V. Koldoba ${ }^{3,4}$, R. \\ Kurosawa $^{5}$, P. S. Lii ${ }^{1}$, and G. V. Ustyugova ${ }^{3}$, \\ ${ }^{1}$ Department of Astronomy, Cornell University, Ithaca, NY 14853-6801, USA \\ ${ }^{2}$ Institut de Recherche en Astrophysique et Planetologie, Toulouse, 31400, France \\ ${ }^{3}$ Keldysh Institute of Applied Mathematics, Moscow, 125047, Russia \\ ${ }^{4}$ Moscow Institute of Physics and Technology, Dolgoprudny, Moscow Region, 141700, Russia \\ ${ }^{5}$ Max-Planck-Institut für Radioastronomie, Auf dem Hügel, 69, 5312 Bonn, Germany
}

\begin{abstract}
We review recent axisymmetric and three-dimensional (3D) magnetohydrodynamic (MHD) numerical simulations of magnetospheric accretion, plasma-field interaction and outflows from the disk-magnetosphere boundary.
\end{abstract}

\section{Introduction}

Dynamically important magnetic fields are present in many classes of accreting stars. Some of them are very young, such as Classical T Tauri stars (CTTSs), while others are very old - white dwarfs and neutron stars. The light-curves from these stars show complex patterns of periodic, quasi-periodic, or irregular variability which can be connected with different processes at the disk-magnetosphere boundary, such as complex paths of matter flow around the magnetosphere, rotations of the hot spots, waves excited in the inner disk, inflation and reconnection of external magnetic field lines of the magnetosphere, and other processes (e.g., [1]). Outflows are observed from a number of CTTSs (e.g., [2]) and a few accreting neutron stars and white dwarfs (e.g., [3]). Some of the outflows may originate at the disk-magnetosphere boundary (e.g., $[4,5])$. The problem of the disk-magnetosphere interaction is multidimensional and requires global axisymmetric and three-dimensional (3D) numerical simulations. Below, we describe results of recent numerical simulations of magnetospheric accretion and outflows from the disk-magnetosphere boundary.

\section{Magnetospheric Accretion}

Magnetospheric accretion is a complex process, where the inner disk matter interacts with the magnetosphere of the star. The result of such an interaction depends on a number of factors, such as the size of the magnetosphere, the period of stellar rotation, and the structure of the magnetic field of the star. Observational properties of magnetized stars also depend on whether or not a star excites waves in the inner disk. Significant progress has been achieved in understanding the disk-magnetosphere interaction due to global 3D simulations performed with the Cubed Sphere code [6, 7]. In particular, it was found that a magnetized star may accrete in either stable or unstable regimes (see Sec. 2.2).

ae-mail: romanova@astro.cornell.edu

This is an Open Access article distributed under the terms of the Creative Commons Attribution License 2.0, which permits unrestricted use, distribution, and reproduction in any medium, provided the original work is properly cited. 

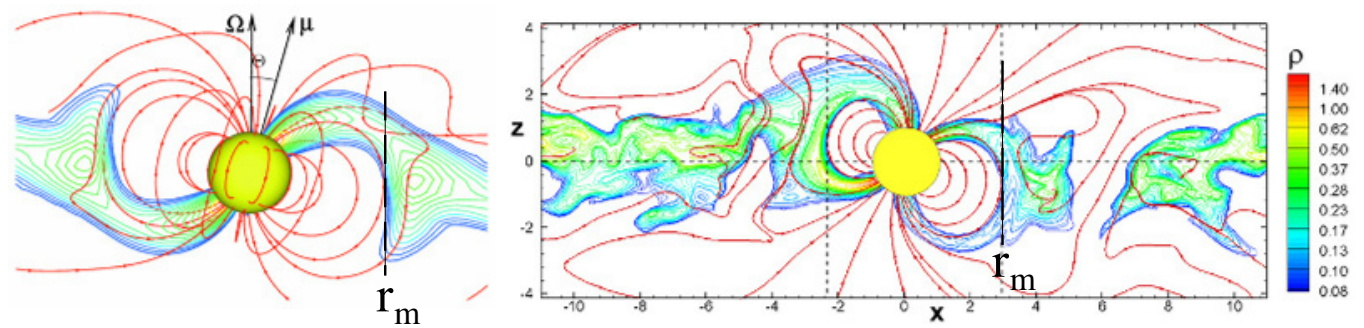

Figure 1. Results of 3D MHD simulations of accretion onto a star with a tilted dipole magnetic field in cases of an $\alpha$-disk (left panel, from [10]) and a turbulent, MRI-driven disk (right panel, from [13]).

It has also become possible to model accretion to stars with complex magnetic fields and to model young stars with realistic parameters (Sec. 2.3). In more recent 3D simulations, accretion to a star with a large magnetosphere and from a very thin disk has been investigated (Sec. 2.4). It has also become possible to investigate waves in the disk excited by a tilted rotating magnetosphere (Sec. 2.5).

\subsection{Truncation of the disk by the magnetosphere}

If the magnetic field of the star is sufficiently strong, then it truncates the accretion disk at a radius $r_{m}$ where the magnetic stress in the magnetosphere matches the matter stress in the disk. In the disk the main stress is connected with the azimuthal components of the stress tensor: $T_{\phi \phi}=\left[p+\rho v_{\phi}^{2}\right]+$ $\left[B^{2} / 8 \pi-B_{\phi}^{2} / 4 \pi\right]$, where $\rho, p, B, B_{\phi}$ are local density, gas pressure, total and azimuthal magnetic field in the disk (we neglect the viscous stress, which is much smaller); $v_{\phi}$ is the azimuthal velocity in the reference frame of the rotating magnetosphere: $v_{\phi}=v_{\text {disk }}-v_{m}$. At the innermost edge of the disk, $B_{\phi}<<B$ and the truncation radius is described by the condition $p+\rho v_{\phi}^{2}=B^{2} / 8 \pi$. We also introduce the modified plasma parameter

$$
\beta_{1}=8 \pi\left(p+\rho v^{2}\right) / B^{2}
$$

which is analogous to the standard plasma parameter $\beta=8 \pi p / B^{2}$, but the ram pressure of the disk matter $\rho v^{2}$ is included, where the total velocity in a thin disk is $v \approx v_{\phi}$. Both axisymmetric and $3 \mathrm{D}$ simulations confirmed that the inner disk truncates at the radius where $\beta_{1} \approx 1$ (e.g., [7-10]) . Fig. 1 shows examples of 3D simulations in the case of a laminar $\alpha$-disk [11] (left panel, from [10]) and a turbulent, MRI-driven [12] disk (right panel, from [13]). We noticed that the condition $\beta=8 \pi p / B^{2}=1$ can also be used, in particular, in cases where $v_{\text {disk }} \approx v_{m}$, and also for finding the position of the funnel streams, which are at slightly larger distances than the inner edge of the disk. [14] derived the magnetospheric radius from different conditions, and found only small difference between different radii $r_{m}$.

The magnetospheric radius has also been derived theoretically in analogy with the Alfvén radius in the case of spherical accretion (e.g., [15]):

$$
r_{m}=k\left[\mu^{4} /\left(\dot{M}^{2} G M_{\star}\right)\right]^{1 / 7}, \quad k \sim 1,
$$

where $\mu=B_{\star} R_{\star}^{3}$ is the magnetic moment of the star with a surface field $B_{\star}, \dot{M}$ is the disk accretion rate, $M_{\star}$ and $R_{\star}$ are the mass and radius of the star. This formula has been tested in a few axisymmetric simulations by [9, 16]. It was found that eqs. 1 and 2 give the same $r_{m}$ if $k \approx 0.5$ [9]. [17] reinvestigated this issue in multiple 3D simulations while taking $\beta=1$ for finding $r_{m}$. It was found that the dependence is slightly different: $r_{m} / R_{\star} \approx 1.06\left[\mu^{4} /\left(\dot{M}^{2} G M_{\star} R_{\star}^{7}\right)\right]^{1 / 10}$, which can be explained by the compression of the magnetosphere and its departure from the dipole shape. 


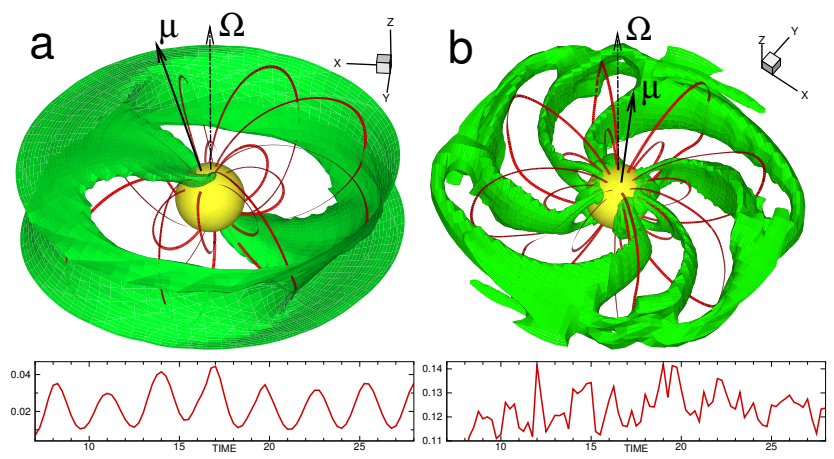

Figure 2. Left panel: a 3D view of matter flow in the stable regime of accretion. Right panel: a 3D view of accretion in the unstable regime. Bottom panels show the light-curves from the hot spots. From [20].

\subsection{Stable and unstable regimes of accretion}

Global 3D simulations of accretion to stars with relatively small magnetospheres (a few stellar radii) show that accreting magnetized stars may be in either stable or unstable regimes of accretion. In the stable regime, matter accretes to the star in two ordered funnel streams and forms two antipodal spots at its surface $[6,7,10]$. Rotation of the star gives an almost sinusoidal light-curve (see Fig. 2, left panel). Such light-curves are observed, e.g., in accreting millisecond pulsars (AMPs) [18] and many CTTSs [19]. In the unstable regime, matter penetrates through the magnetosphere in chaotic equatorial "tongues" due to the magnetic Rayleigh-Taylor (R-T) instability [20, 21], and the light-curve from the hot spots may be chaotic (see Fig. 2, right panels). Chaotic light-curves with no clear periods are observed in many CTTSs [19]. In the intermediate regime, both the magnetospheric funnel and the unstable tongues are observed [22, 23]. Earlier, [24] suggested that the R-T and Kelvin-Helmholtz instabilities may be efficient mechanisms of mixing accreting matter to magnetosphere (see also [25]). Simulations show that the unstable regime may lead to a qualitatively different state of accretion.

Observational properties of CTTSs in stable and unstable regimes have been studied by [26, 27], where the cubed sphere grid of the 3D MHD code has been projected to the adaptive mesh refinement grid of 3D radiative transfer code Torus [28], and time-dependent hydrogen spectral lines were calculated from 3D MHD modeled flow. These 3D+3D simulations have shown that in the stable regime, periodic obscuration of stellar light by a funnel stream (where matter moves away from the observer) produces periodic variability of the redshifted wing of spectral lines, while in the unstable regime (where several tongues are usually present in the field of view of the observer), the redshifted absorption is quasi-steady.

The boundary between stable and unstable regimes has been investigated by [20,21]. They found that at a fixed period of the star, the boundary depends on the accretion rate: accretion becomes unstable at higher accretion rates. The accretion rate has been varied using the $\alpha$-parameter of viscosity [11]. In more recent simulations, performed at a higher grid resolution and a small $\alpha=0.02$ [29], the period of the star has been varied, and a simple criterion has been derived: the accretion is unstable if $r_{c} \gtrsim 1.4 r_{m}$, where $r_{c}=\left(G M_{\star} / \Omega_{\star}^{2}\right)^{1 / 3}$ is the corotation radius, and $\Omega_{\star}$ is the angular velocity of the star. In both investigations, the boundary between the regimes agrees with the theoretical study by [30].

In the cases of very small magnetospheres, $r_{m} \lesssim(1-2) R_{\star}$, unstable accretion becomes more ordered, with one or two unstable tongues forming and rotating with the angular velocity of the inner disk. This may cause the frequency of the inner disk to dominate in the spectrum [31]. The typical 

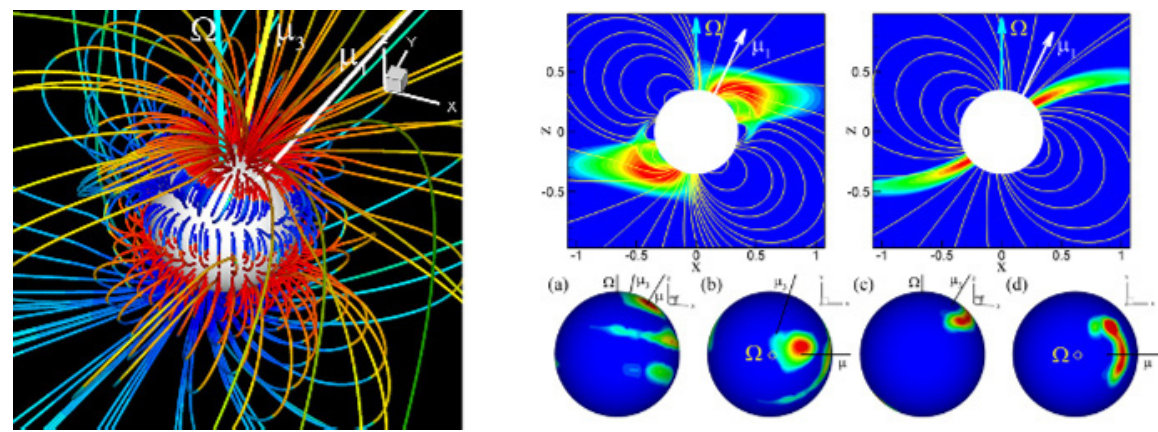

Figure 3. Left panel: a 3D view of the initial magnetic field in a 3D MHD model of a young star V2129 Oph, where the magnetic field is dominated by a $0.35 \mathrm{kG}$ dipole and $1.2 \mathrm{kG}$ octupole components. Right panel: slices of the density distribution and hot spots (energy distribution at the surface of the star) in cases of the dipole+octupole field and a pure dipole field. From [42].

feature of this frequency is that it varies in time: it increases with accretion rate, when the inner disk moves inwards. In CTTSs, this frequency can be mistaken for the frequency of the star, or may lead to the phenomenon of a drifting "period", observed in some CTTSs (e.g., [32]). In AMPs, two highfrequency QPOs are observed [33]. The upper frequency, $v_{u}$, may be connected with the rotation of the unstable tongues. The lower frequency, $v_{l}$, may be connected with the rotation of a funnel stream about the magnetic pole, which is possible in stars with very small tilts of the magnetosphere [23]. Both the funnels and the tongues produce hot spots on the surface of the star which rotate with similar frequencies. Simulations show that both frequencies vary strongly with accretion rate. However, their difference, $v_{u}-v_{l}$, does not vary much and does not correlate with the frequency of the star, which is typical for a number of AMPs [34]. The phenomenon of rotating funnels and tongues is probably determined or enhanced by waves in the inner disk (see Sec. 2.5).

\subsection{Accretion to stars with complex magnetic field}

Stars may have a complex magnetic field (e.g., [35-37]). The magnetic field can be decomposed into a superposition of tilted dipole, quadrupole, octupole and higher order multipoles: $\mathbf{B}_{\star}=\mathbf{B}_{\text {dip }}+\mathbf{B}_{\text {quad }}+$ $\mathbf{B}_{\text {oct }}+\ldots$. Global 3D simulations show that if the magnetic field of a star has dipole and quadrupole field components, then matter partially accretes to the regions of the magnetic poles, and partially to the ring associated with the quadrupole component of the field $[38,39]$. In the case of a superposition of dipole and octupole fields, matter partially flows to two octupole rings [40]. It is often the case that the dipole component dominates at large distances from the star and determines the disk-magnetosphere interaction and the initial path of the funnel streams, while the quadrupole or octupole components determine matter flow close to the star and influence the shapes of the hot spots. Recent measurements of the surface magnetic field in CTTSs have shown that in several stars the dominant components of the field are dipole and octupole, while quadrupole and higher-order components are smaller [35, 41]. This helped us model the magnetospheric accretion to CTTSs with realistic parameters: V2129 Oph, where the octupole component is relatively large [42], and BP Tau, where the octupole component is small [43]. Hot spots derived from simulations were in good agreement with the spots obtained from polarimetric observations and analyses [35, 41].

More recently, a time-variable spectrum in hydrogen lines has been calculated from the 3D MHD model of V2129 and has been compared with the time-variable spectrum obtained from the observations of this star [44]. Comparisons show a good match between the observed and modeled spectra. 

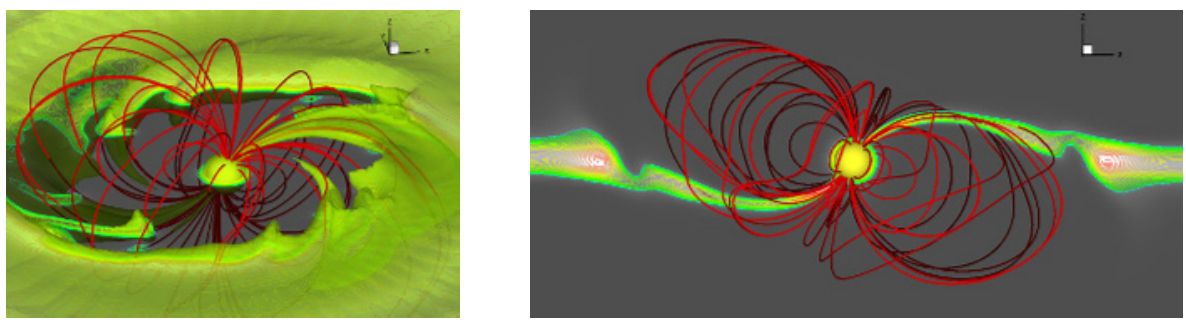

Figure 4. Left panel: a 3D view of matter flow in the case of a star with a relatively large magnetosphere $\left(r_{m} \approx 12 R_{\star}\right)$. Right panel: an XZ-slice of density distribution and sample field lines.

\subsection{Larger magnetosphere, thinner disk}

Earlier simulations are relevant to CTTSs and AMPs, where the size of the magnetosphere is a few times larger than the radius of the star. However, in intermediate polars $r_{m} / R_{\star} \gtrsim 10$, while in X-ray pulsars $r_{m}$ may reach hundreds of stellar radii. More recent simulations were aimed at investigating the disk-magnetosphere interaction in cases of larger magnetospheres, where the magnetospheric radius $r_{m}>10 R_{\star}$ (Romanova et al., in prep). Preliminary simulations show that matter of the inner disk penetrates through the external layers of the magnetosphere up to the depth of 1-2 stellar radii (see Fig. 4). Later on, a few funnel streams form in regions of enhanced density, which coincide with unstable tongues. It is often the case that a few funnel streams form because a few unstable tongues are located in the region of the disk where accretion through funnel streams is favorable. In current simulations, we see that accretion through R-T instability does not lead to the globally-chaotic accretion, as in the case of small magnetospheres. However, this result should be checked+ at a wider range of parameters.

In another set of numerical experiments, the grid was compressed towards the disk, and accretion from a very thin disk $(h / r \approx 0.02-0.03)$ was investigated. The main goal was to understand how matter of the inner disk "climbs" to the magnetospheric wall from the equatorial plane without a sufficient pressure gradient or other forces lifting matter above the equatorial plane. Simulations were done for a relatively small magnetosphere of $\sim 5 R_{\star}$, and the tilt of the dipole was $\Theta=20^{\circ}$. Simulations have shown an interesting phenomenon. The tilted dipole excited bending waves in the disk, which helped lift matter from the equatorial plane to larger heights, from where the gravitational force pulled matter towards the star. This mechanism of lifting may be important in cases of much larger magnetospheres, as in intermediate polars and X-ray pulsars.

\subsection{Warps and waves in the disk}

A tilted magnetosphere applies force to the inner parts of the disk and excites waves in the disk $[45,46]$. If the rotational axis of the star is aligned with the rotational axis of the disk, then waves in the disk are excited on the time-scale of stellar rotation. Global 3D simulations show that when the magnetosphere and the inner disk are in approximate corotation (that is, $r_{m} \approx r_{c}$ ), a large bending wave is formed and rotates with the angular velocity of the star [47]. The height of the wave depends on the density level of the warp and is approximately $(0.1-0.2) R_{\star}$. This result is in agreement with the theoretical findings of [48]. This wave may obscure the light of the star and lead to a special type of variability, with regular dips in the light-curves of CTTSs $[49,50]$. In the cases where the magnetosphere rotates more slowly than the inner disk $\left(r_{m}<r_{c}\right)$, two types of waves are observed in the inner disk. One is a radially-trapped density wave, which appears in the region of the maximum of angular velocity in the inner disk, where its rotation rate varies from Keplerian to that of the star [51]. The other one is an inner bending wave which rotates with near-Keplerian velocity. Both frequencies 

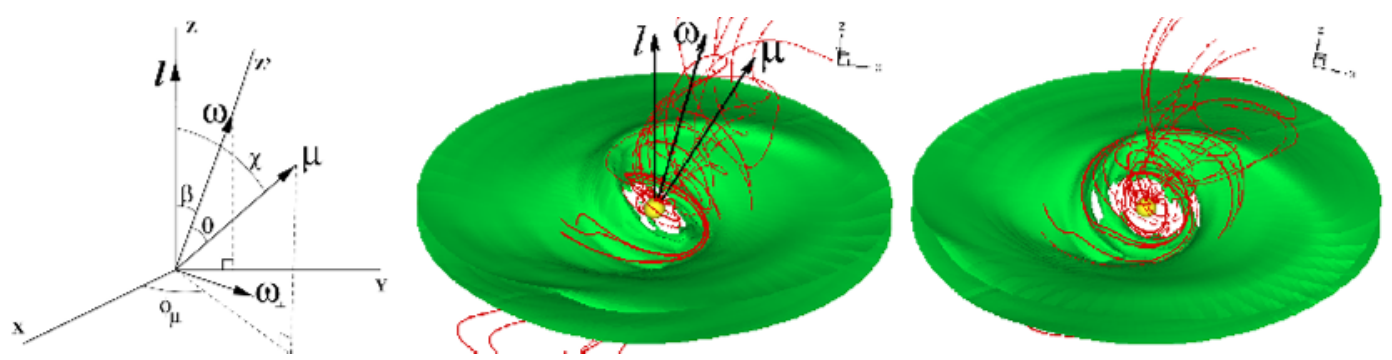

Figure 5. Results of $3 \mathrm{D}$ simulations of disk accretion to a star where both the rotational $(\omega)$ and the magnetic $(\mu)$ axes are tilted with respect to the rotational axis of the disk.

depend on the position of the inner disk and vary with accretion rate [47]. These two waves may also explain the twin-peak QPOs observed in AMPs: the trapped density wave may be responsible for the strongest (lower) QPO frequency, while the inner bending wave may be responsible for the upper QPO. Again, the difference $v_{u}-v_{l}$ does not correlate with the frequency of the star.

If the rotational axis of the star is tilted with respect to the rotational axis of the disk, then the magnetic force acts systematically on the inner disk, pushing it to tilt and to precess [45, 46]. Experimental numerical simulations (Romanova et al., in prep) show that the inner disk is tilted and precesses slowly (see Fig. 5). In this case, the warp has a high amplitude and can obscure or reflect light of the star on the time-scale of the precession. Simulations also show that such a tilt of the inner disk influences the magnetospheric accretion, in particular, the location of the funnel streams and hot spots on the surface of the star. A precessing inner disk may also reflect light from the star and provide low-frequency oscillations, or a modulation of light with the frequency of the precession. This mechanism may explain some of the low-frequency oscillations observed in different magnetized stars.

\section{Outflows from the disk-magnetosphere boundary}

At the disk-magnetosphere boundary, the conditions may be favorable for driving outflows. Matter of the inner disk may be redirected to the outflows if the centrifugal and/or magnetic pressure forces overcome the gravitational force and the magnetic tension force of the closed magnetic field lines of the magnetosphere. The differential rotation of the field lines connecting the star and the disk often leads to inflation and opening of these field lines, which is favorable for outflows. Different regimes of outflows are possible. If the magnetosphere rotates more rapidly than the inner disk (that is, $r_{m}>r_{c}$ ), then the star is in the propeller regime $[52,53]$, where the centrifugal force may drive outflows. On the other hand, if the star rotates more slowly than the inner disk, then the outflows may be driven by the magnetic force. Here, we consider these two types of outflows.

\subsection{Propeller-driven Winds and Jets}

Axisymmetric simulations show that in the propeller regime a significant fraction of the disk mass can be redirected to the outflows by the rapidly-rotating magnetosphere [54-56]. In this regime, accretion and outflows occur in cycles, where matter accumulates in the inner disk, diffuses across the field lines of the rapidly-rotating magnetosphere, and is ejected to the outflows; then, the magnetosphere expands and the cycle repeats (see also [57-59]). Most of the matter flows to a conically-shaped wind, which is only gradually collimated with distance [55, 58] (see Fig. 6). In addition, a smaller amount of matter flows to a magnetically-dominated, well-collimated Poynting jet [60], where matter is accelerated rapidly by the magnetic force [61]. The jet carries significant energy and angular momentum out of 

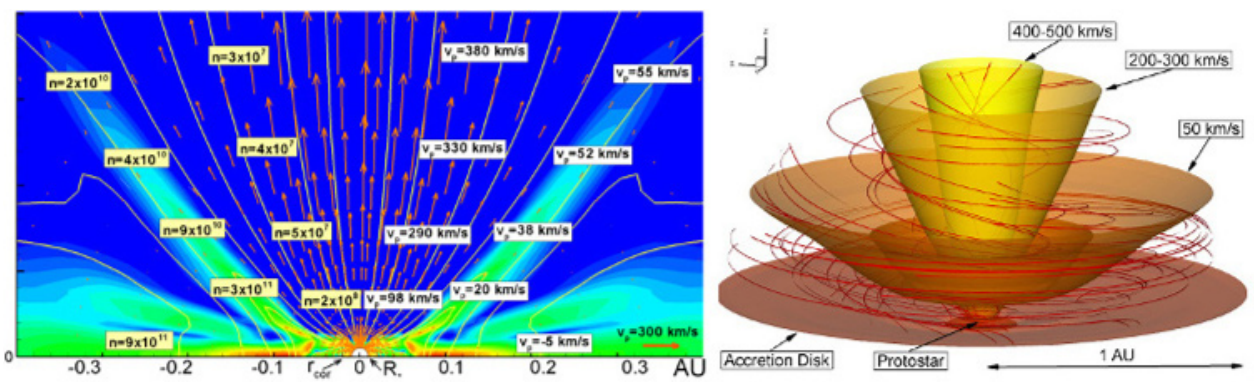

Figure 6. Left panel: density and velocity distribution in the propeller regime with numbers corresponding to CTTSs [55]. Right Panel: 3D rendering shows magnetic field lines and density levels corresponding to different velocities.

the star, causing it to spin down (e.g., [54]). Part of the angular momentum is transferred from the star to the partially-inflated field lines $[16,58]$. In CTTSs, the propeller mechanism may be responsible for their spinning down from near break-up speed to less than $10 \%$ of this speed (e.g., [54]). In AMP SAX J1808.4-3658, 1Hz flaring oscillations have been observed at the end of an outburst [62], and may be connected with the propeller regime $[63,64]$. A similar phenomenon has been observed in a few cataclysmic variables, e.g., in AE Aqr (e.g., [65]).

\subsection{Conical Winds}

Recent numerical simulations show a new type of winds which can be important during episodes of enhanced accretion rate, as during the outbursts of accretion in AMPs, or in young EXOR and FUOR type stars [55]. Simulations show that the newly-incoming matter compresses the magnetosphere of the star, the field lines inflate due to the differential rotation between the disk and the star, and conically-shaped winds flow out of the inner disk [55, 66]. These winds are driven by the magnetic force, $F_{M} \propto-\nabla\left(r B_{\phi}\right)^{2}$, which arises due to the wrapping of the field lines above the disk [61]. The wind is also gradually collimated by the magnetic hoop-stress, and can be strongly collimated in the cases of high accretion rates [67]. A rapid rotation of the star is not required. Moreover, the star can rotate much more slowly than the inner disk (at $r_{m}<<r_{c}$ ), which may be typical during accretion outbursts in different types of stars. This is different from the X-winds, which require the condition $r_{m} \approx r_{c}$. Conical winds may appear during an outburst of accretion and continue for the entire duration of the outburst. A magnetic field of a few $\mathrm{kG}$ is required for FUORs, while in EXORs a and CTTSs the field can be weaker. The conical wind model has been applied to FU Ori and compared with the empirical model based on the spectral analysis of the winds in FU Ori [68]. A reasonably good agreement was found between these models [69].

Observational properties of conical winds in application to CTTSs were investigated in [66, 70], where the spectrum in $\mathrm{He}$ and $\mathrm{H}$ lines was calculated using the Torus code. Simulations show that conical winds produce a narrow blue absorption component in the spectrum (see Fig. 7). Such a blue component is frequently observed in the spectra of CTTSs (e.g., [71]).

\subsection{Asymmetric and one-sided outflows}

In stars with a complex magnetic field, outflows may be asymmetric due to the top-bottom asymmetry of the magnetic field. For example, the superposition of an axisymmetric dipole and a quadrupole leads to a magnetic configuration where the magnetic flux is larger on one side of the equatorial plane and smaller on the other side (see Fig. 8, right panel). Axisymmetric simulations of the propeller regime show that stronger outflows are observed on the side where the magnetic flux is larger (see 

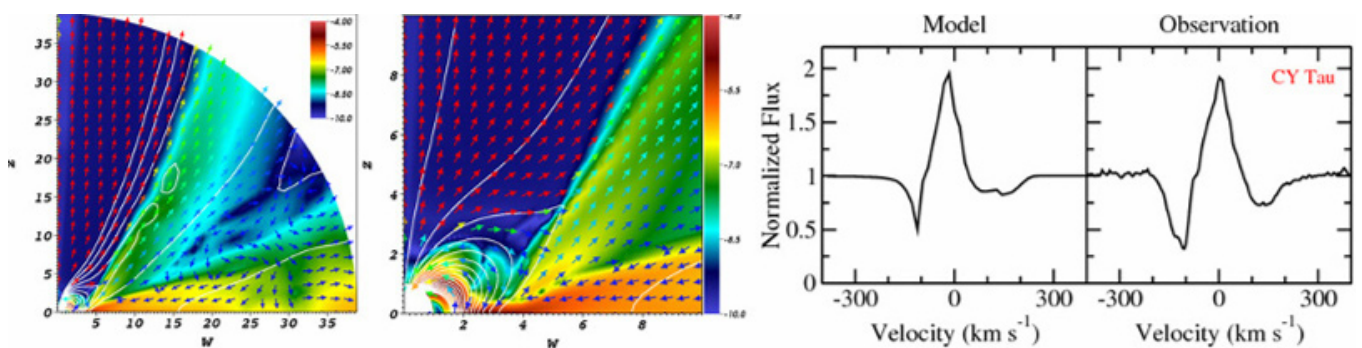

Figure 7. Left two panels: axisymmetric simulations of conical winds, shown on larger and smaller scales. Right two panels: a comparison of modeled and observed spectra in CTTS CY Tau. From [66].

left panel of Fig. 8). In this case, the matter and energy fluxes will be systematically higher in one direction, and lower in the other direction. One-sided outflows are observed in a number of young stars [72].

Axisymmetric simulations of the entire region also show that even in the case of a pure dipole field, outflows are usually one-sided. However, the direction of the outflows switches frequently, and therefore the averaged matter and energy fluxes of the outflows above and below the equatorial plane are expected to be approximately equal in both directions [73]. Recent simulations of the propeller regime in the case of MRI-driven accretion have also shown that the outflows are one-sided, but the wind switches sides much less frequently [58].
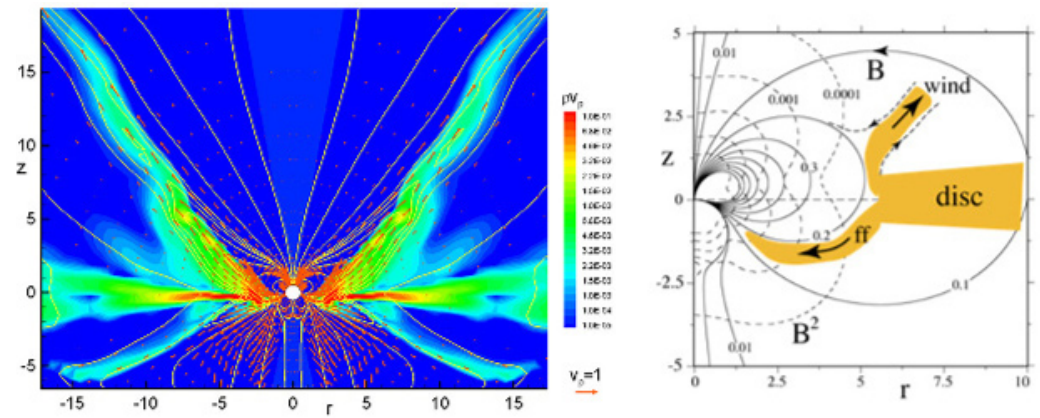

Figure 8. Left panel: a density slice and sample field lines show the result of an axisymmetric simulation of accretion to a star with dipole and quadrupole fields. Right panel: a sketch of accretion and outflows (from [73]).

\section{Disk-magnetosphere interaction and variability}

Here, we briefly discuss the different variabilities expected during the disk-magnetosphere interaction. In the stable regime, the hot spots rotate approximately with the period of the star, although variation of the accretion rate leads to phase-shifts of spots in the azimuthal direction: the phase increases with accretion rate $[10,47]$. This may lead to the phenomenon of a drifting period in CTTSs, where the period is often derived from the observations of hot spots. In AMPs, the variation of phase with luminosity has been observed (e.g., [74]). Variation of phase may lead to the observed gradual variation of the "period" of the star, which is determined from the observations of hot spots. In the unstable regime, accretion is often stochastic, with a time-scale of a few accretion events per period of the inner disk. However, the entire set of unstable tongues rotates with the angular velocity of the inner disk, and this frequency may be seen in the power spectra. The period of the star can also be seen, unless 
the instability is very strong $[22,23]$. In the cases of very small magnetospheres, $r_{m} \lesssim 2 R_{\star}$, one or two unstable tongues dominate and rotate with the frequency of the inner disk [55]. In the case of CTTSs, this period can be mistakenly interpreted as the strongly drifting "period" of the star. The rotation of the foot-points of moving funnel streams and unstable tongues may be determined or enhanced by waves in the inner disk.

Longer time variability (a few periods of the inner disk or more) can be connected with the opening and reconnection of the external field lines of the magnetosphere [75]. This phenomenon has been observed in the conical wind model [55, 67] and in the Magnetospheric Ejections model [16]. These oscillations may have very long periods in the cases of very low diffusivity. If the rotational axes of the star and the disk are misaligned, then a slow precession of the inner disk is expected ([45], Sec. 2.5), and this may be a source of persistent low-frequency oscillations. Another type of low-frequency oscillations may be connected with episodic accumulation and accretion of matter in a weak propeller regime [59].

\section{Acknowledgments}

We thank the conference organizers, especially Dr. E. Bozzo, for the excellent meeting. MMR is grateful for the generous support of her trip. Resources supporting this work were provided by the NASA High-End Computing (HEC). The research was supported in part by NASA grant NNX11AF33G and NSF grant AST-1211318. GVU and AVK were supported in part by FAP14.B37.21.0915, SS-1434.2012.2, and RFBR 12-01-00606-a.

\section{References}

[1] T.J. Harries, MNRAS 315, 722 (2000)

\section{References}

[1] Bouvier, J., Alencar, S. H. P., Harries, T. J., Johns-Krull, C. M., Romanova, M. M., Protostars and Planets V, B. Reipurth, D. Jewitt, and K. Keil (eds.), (University of Arizona Press, Tucson, 2007), p. 479

[2] Ray, T., Dougados, C., Bacciotti, F., Eislöffel, J., Chrysostomou, A., Protostars and Planets V, B. Reipurth, D. Jewitt, and K. Keil (eds.), (University of Arizona Press, Tucson, 2007) p. 231

[3] Fender, R.P., Nature 427, 222 (2004)

[4] Shu, F., Najita, J., Ostriker, E., Wilkin, F., Ruden, S., Lizano, S., ApJ 429, 781 (1994)

[5] Ferreira, J., Dougados, C., Cabrit, S., A\&A 453, 785 (2006)

[6] Koldoba, A. V., Romanova, M. M., Ustyugova, G. V., Lovelace, R. V. E., ApJ 576, L53 (2002)

[7] Romanova, M.M., Ustyugova, G.V., Koldoba, A.V., Lovelace, R.V.E. 2003, ApJ 595, 1009 (2003)

[8] Romanova, M.M., Ustyugova, G.V., Koldoba, A.V., \& Lovelace, R.V.E., ApJ 578, 420 (2002)

[9] Long, M., Romanova, M.M., Lovelace, R.V.E., ApJ 634, 1214 (2005)

[10] Romanova, M.M., Ustyugova, G.V., Koldoba, A.V., Lovelace, R.V.E., ApJ 610, 920 (2004)

[11] Shakura, N.I., \& Sunyaev, R.A., A\&A 24, 337 (1973)

[12] Balbus, S.A., \& Hawley, J.F., ApJ 376, 214 (1991)

[13] Romanova, M.M., Ustyugova, G.V., Koldoba, A.V., Lovelace, R.V.E., MNRAS 421, 63 (2012)

[14] Bessolaz N., Zanni C., Ferreira J., Keppens R., Bouvier J., A\&A 478, 155 (2008) 
[15] Lamb, F. K., Pethick, C. J., Pines, D., ApJ, 184, 271 (1973)

[16] Zanni, C., \& Ferreira, J., A\&A 550, A99 (2013)

[17] Kulkarni, A., \& Romanova, M.M., MNRAS 433, 3048 (2013)

[18] Ibragimov, A., \& Poutanen, J., MNRAS 400, 492

[19] Herbst, W., Herbst, D. K., Grossman, E. J., Weinstein, D. 1994, AJ 108, 1906

[20] Romanova, M.M., Kulkarni, A.K., Lovelace, R.V.E., ApJ 673, L171 (2008)

[21] Kulkarni, A., \& Romanova, M.M., MNRAS 386, 673 (2008)

[22] Kulkarni, A., \& Romanova, M.M., MNRAS 386, 673 (2009)

[23] Bachetti, M., Romanova, M.M., Kulkarni, A., Burderi, L., di Salvo, T., MNRAS 403, 1193 (2010)

[24] Arons, J., \& Lea, S.M., ApJ 207, 914 (1976)

[25] Lovelace, R.V.E., Romanova, M.M., Newman, W.I., MNRAS, 402, 2575 (2010)

[26] Kurosawa, R, Romanova, M.M., Harries, T.J., MNRAS 385, 1931 (2008)

[27] Kurosawa, R., \& Romanova, M.M., MNRAS 431, 2673 (2013)

[28] Harries, T.J., MNRAS 315, 722 (2000)

[29] Blinova, A.A., Romanova, M.M., \& Lovelace, R.V.E., Physics at the Magnetspheric Boundary (Geneva, Switzerland, 2013) (arXiv:1309.4363)

[30] Spruit H. C., Stehle R., Papaloizou J. C. B., MNRAS 275, 1223 (1995)

[31] Romanova, M.M., \& Kulkarni, A.K., ApJ 398, 1105 (2009)

[32] Rucinski, S.M., et al. MNRAS 391, 1913 (2008)

[33] van der Klis, M., Compact Stellar X-Ray Sources, Eds. W.H.G. Lewin \& M. van der Klis (Cambridge: Cambridge Univ. Press, 2006), p. 39

[34] Belloni, T., Méndez, M., Homan, J., MNRAS 376, 1133 (2007)

[35] Donati, J.-F., Jardine, M. M., Gregory, S. G., et al., MNRAS 380, 1297 (2007)

[36] Johns-Krull, C. M., ApJ 664, 975 (2007)

[37] Gregory, S. G. 2011, American Journal of Physics 79, 461

[38] Long, M., Romanova, M.M., \& Lovelace, R.V.E., MNRAS, 374, 436 (2007)

[39] Long, M., Romanova, M.M., \& Lovelace, R.V.E., MNRAS, 386, 1274 (2008)

[40] Long, M., Romanova, M.M., Lamb, F.K., New Astronomy 17, 232 (2012)

[41] Donati J.-F., Jardine M. M., Gregory S. G. et al., MNRAS 386, 1234 (2008)

[42] Romanova, M. M., Long, M., Lamb, F. K., Kulkarni, A. K., Donati, J.-F., MNRAS, 411, 915 (2011)

[43] Long, M., Romanova, M.M., Kulkarni, A.K., Donati, J.-F., MNRAS 413, 1061 (2011)

[44] Alencar S.H.P., Bouvier, J, Walter, F.M., Dougados, C., Donati, J.F., Kurosawa, R., Romanova, M.M. et al., A\&A 541, A116 (2012)

[45] Lai, D., ApJ 524, 1030 (1999)

[46] Lipunov, V.M., \& Shakura, N.I., Soviet Astron. Let. 6, 14 (1980)

[47] Romanova, M.M., Ustyugova, G.V., Koldoba, A.V., Lovelace, R.V.E., MNRAS 430, 699 (2013)

[48] Terquem, C., \& Papaloizou, J.C.B., A\&A 360, 1031 (2000)

[49] Alencar, S.H.P. et al., A\&A 519, A88 (2010)

[50] Bouvier J. et al., A\&A 349, 619 (1999)

[51] Lovelace, R. V. E. \& Romanova, M. M., ApJ 670, L13 (2007)

[52] Illarionov, A. F., \& Sunyaev, R. A., A\&A, 39, 185 (1975)

[53] Lovelace, R.V.E., Romanova, M.M., Bisnovatyi-Kogan, G.S., ApJ, 514, 368 (1999) 
[54] Romanova, M.M., Ustyugova, G.V., Koldoba, A.V., Lovelace, R.V.E., ApJ 635, 165L (2005)

[55] Romanova, M.M., Ustyugova, G.V., Koldoba, A.V., Lovelace, R.V.E., MNRAS 399, 1802 (2009)

[56] Ustyugova, G.V., Koldoba, A.V., Romanova, M.M., Lovelace, R.V.E., ApJ 646, 304 (2006)

[57] Goodson, A.P., Winglee, R. M., Böhm, K.-H., ApJ 489, 199 (1997)

[58] Lii, P.S., Romanova, M.M., Ustyugova, G.V., Koldoba, A.V., Lovelace, R.V.E. MNRAS, in press (arXiv:1304.2703) (2013)

[59] D’Angelo C. R., \& Spruit H. C., MNRAS 406, 1208 (2010)

[60] Lovelace, R.V.E., Li, H., Koldoba, A.V., Ustyugova, G.V., Romanova, M.M., ApJ 572, 445 (2002)

[61] Lovelace, R.V.E., Berk, H.L., Contopoulos, J., ApJ 379, 696 (1991)

[62] van Straaten, S., van der Klis, M., Wijnands, R., ApJ 619, 455 (2005)

[63] Patruno, A., Watts, A.L., Klein-Walt, M., Wijnands, R., van der Klis, M., ApJ 707, 1296

[64] Patruno, A., \& D’Angelo, C., ApJ 771, 94 (2013)

[65] Mauche, C.W., MNRAS 369, 1983 (2006)

[66] Kurosawa, R., \& Romanova, M.M., MNRAS 426, 2901 (2012)

[67] Lii, P.S., Romanova, M.M., Ustyugova, G.V., Koldoba, A.V., Lovelace, R.V.E. MNRAS 420, 2020 (2012)

[68] Calvet N., Hartmann L., Kenyon S. J., ApJ 402, 623 (1993)

[69] Königl, A., Romanova, M.M., Lovelace, R.V.E., MNRAS 416, 757 (2011)

[70] Kurosawa, R., Romanova, M.M., Harries, T.J., MNRAS 416, 2623 (2011)

[71] Edwards, S., Fischer, W., Hillenbrand, L., Kwan, J., ApJ 646, 319 (2006)

[72] Bacciotti, F., Eisloffel, J., Ray, T.P., A\&A 350, 917 (1999)

[73] Lovelace, R.V.E., Romanova, M.M., Ustyugova, G.V., Koldoba, A.V., MNRAS 408, 2083 (2010)

[74] Papitto, A., di Salvo, T., Burderi, L., Menna, M. T., Lavagetto, G., Riggio, A., MNRAS 375, 971 (2007)

[75] Aly, J. J., \& Kuijpers, J., A\&A 227, 473 (1990) 